This document is confidential and is proprietary to the American Chemical Society and its authors. Do not copy or disclose without written permission. If you have received this item in error, notify the sender and delete all copies.

\title{
Synthesis of an Amphiphilic Miktoarm Star Terpolymer for Self-Assembly into Patchy Polymersomes
}

\begin{tabular}{|r|l|}
\hline Journal: & ACS Macro Letters \\
\hline Manuscript ID & mz-2015-00913n.R1 \\
\hline Manuscript Type: & Letter \\
\hline Date Submitted by the Author: & 12 -Jan-2016 \\
\hline Complete List of Authors: & $\begin{array}{l}\text { Gaitzsch, Jens; University of Basel, Chemistry } \\
\text { Chudasama, Vijay; Department of Chemistry and Biochemistry, University } \\
\text { College London } \\
\text { Morecroft, Eloise; Department of Chemistry and Biochemistry, University } \\
\text { College London } \\
\text { Messager, Lea; Department of Chemistry and Biochemistry, University } \\
\text { College London } \\
\text { Battaglia, Giuseppe; Department of Chemistry and Biochemistry, University } \\
\text { College London }\end{array}$ \\
\hline
\end{tabular}

SCHOLARONE

Manuscripts 
In developing cutting-edge technologies, scientists have often tried to mimic and add value to biological systems derived from nature. In the area of nanotechnology, the mimicking and improving of cells or cellular compartments has come into focus. ${ }^{1,2}$ In this respect, the formation of polymersomes from amphiphilic block copolymers is an important step as they represent a synthetic equivalent to natural compartments (liposomes) typically formed by phospholipids. ${ }^{3-6}$ Similar to liposomes, polymersomes are vesicular and emerge from the formation of an amphiphilic membrane that encloses around a given aqueous volume. The synthetic and macromolecular nature of copolymers enables the synthesis of polymersomes with significantly increased chemical variety as well as improved chemical and mechanical stability. ${ }^{7-11}$ It is, for example, possible to trigger polymersome disassembly by a specific external trigger, such as light, ${ }^{12}$ temperature ${ }^{13,14}$ or $\mathrm{pH}^{15,16}$ amongst others ${ }^{17}$. The latter is of importance for drug-delivery purposes due to the reduction in $\mathrm{pH}$ upon cellular uptake via any endocytic pathway. ${ }^{18}$ From the $\mathrm{pH}$ sensitive polymers available, it is known that poly(diisopropylaminoethyl methacrylate) (PDPA, Figure 1) is best suited for this purpose. ${ }^{5,6,15}$ From the many hydrophilic polymers available, poly(ethylene glycol) (PEG, Figure 1$)^{19,20}$ and poly(methacryl phosphoryl choline) (PMPC, Figure 1) are commonly used for polymersomes as they are employed clinically with no toxicity issues. ${ }^{6,21,22}$ Additionally, we recently reported that PMPC containing polymersomes enter cells via a class of receptors known as scavenger receptor B and these are overexposed in some cancer cells. ${ }^{23}$ We also observed that cellular uptake can be considerably enhanced when PMPC-PDPA is mixed with cell inert PEG-PDPA to form polymersomes with a patterned surface, or patchy polymersomes, as both polymers phase separate on the vesicle surface ${ }^{18,24,25}$ However, the formation of patchy polymersomes by mixing two diblock copolymers leads to a slow yet full phase separation. ${ }^{25,26}$ The use of a lineactant, which contains all three polymers, is a feasible option to stabilize the patches. In fact, the patches would already form if the pure lineactant is mixed with PMPC-PDPA, eliminating the need for a more complex ternary mixture. An example of a simple lineactant would be a linear triblock-copolymer. Howev- er, linear $A B C$ triblock copolymers form membranes with two possible conformations: either bridging across where $A$ and $C$ are located on opposite sides of the membrane, or looping where $A$ and $C$ are located on the same side of the membrane. ${ }^{5,27,28}$ In order to fix the conformation, the polymer arms need to be linked at a single point to form a miktoarm star terpolymer. This kind of polymer is already known to self-assemble into vesicles. ${ }^{29-32}$ It was therefore our goal to synthesise an amphiphilic miktoarm starpolymer to be mixed with PMPC-PDPA to control the surface topology of patchy polymersomes. ${ }^{33,34}$

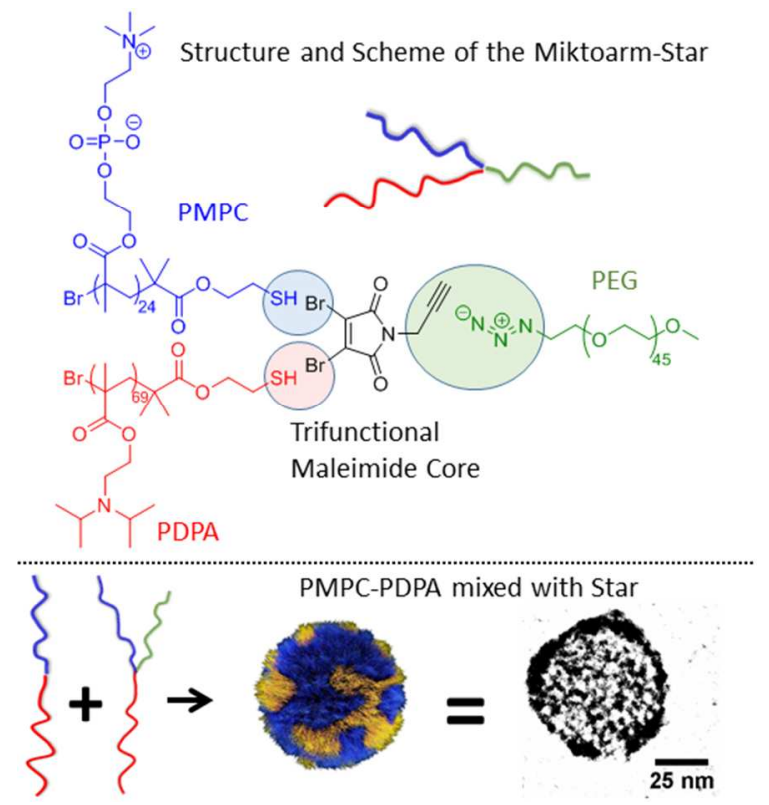

Figure 1: Scheme of the miktoarm star, including the click reactions used to synthesise it. PMPC (blue) and PDPA (red) are attached using thiol/dibromomaleimide conjugation, while PEG (green (structure) + yellow (vesicle)) is clicked on using CuAAC chemistry. If self-assembled together with a PMPC-PDPA diblock, patchy polymersomes evolve.

In this article, we disclose the formation of a miktoarm star terpolymer consisting of PEG, PMPC and PDPA (Figure 1). All polymers were grafted onto a central moiety, using efficient, highly tolerant reactions to obviate any issues arising from poor reaction efficiency due to steric 
hindrance. The self-assembly behaviour of the completed miktoarm star on its own and in a mixture with a linear PMPC-PDPA diblock was then evaluated, with particular focus on surface topology.

Our study began with the selection of a suitable core moiety that would be amenable miktoarm star terpolymer synthesis. This meant a molecule with three appropriately reactive sites to allow step-wise attachment of the three different prepolymers in a highly efficient and controlled manner. In this respect, $N$-functionalised dibromomalimides, previously reported by Baker, Caddick and coworkers were a promising starting point. ${ }^{35,36}$ According to previous studies, the bromine moieties can be substituted sequentially by thiols in a controlled and efficient manner (Figure 1). With respect to the third point of attachment, two different approaches were considered: a) reaction of an amine (e.g. PEG-NH2) with a carbamate-activated dibromomaleimide, ${ }^{35}$ and b) use of an $N$-alkyne functionalised dibromomaleimide, which could undergo a Copper catalysed Azide-Alkyne Click (CuAAC) reaction (Figures 1 and 2). ${ }^{36,37}$ As many PEG derivatives are commercially available, the corresponding amine and azide could be purchased.

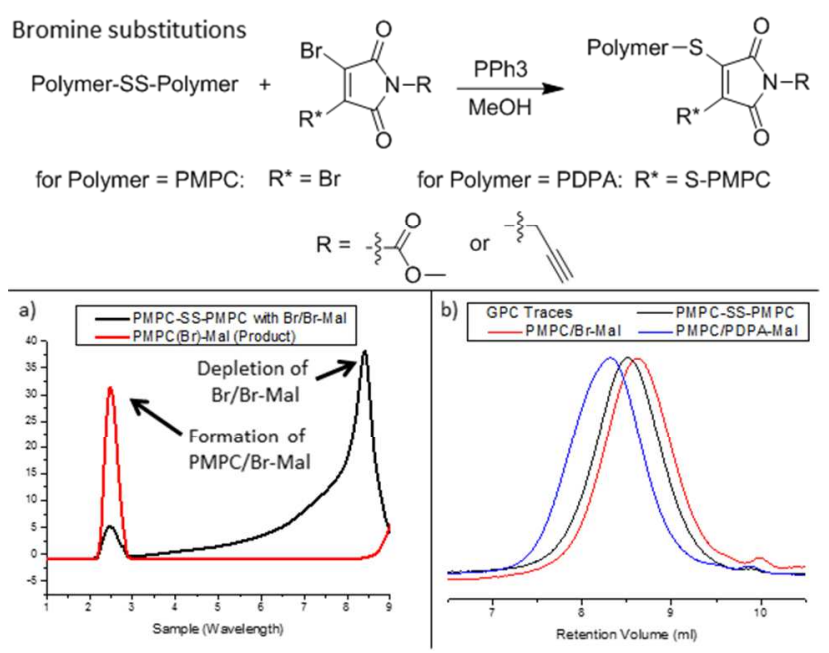

Figure 2: Thiol/Bromine substitution reaction to attach PMPC and PDPA onto the Maleimide core. (a) Complete consumption of the $\mathrm{Br} / \mathrm{Br}-\mathrm{Mal}$ is confirmed by HPLC while (b) GPC shows the complete consumption of the PMPC-SSPMPC towards PMPC/Br-Mal (product is smaller) and complete conversion towards the larger PMPC/PDPA-Mal with no shoulder in any case.

The carbamate activated and alkyne bearing dibromomaleimides were synthesised via the routes reported previously (see SI for details). With both core maleimide molecules in-hand, the synthesis of the miktoram star terpolymer with the corresponding prepolymers was appraised. Thus we turned our attention to how we would incorporate a thiol moiety into PMPC and PDPA respectively (see Figure 1). Both methacrylates polymers were synthesised using a commercially available bifunctional ATRP initiator, containing a central disulphide bond. ${ }^{26}$ In accordance with previously published results, a PMPC of 25 repeating units and a PDPA of 70 repeating units was targeted to match the PMPC25-PDPA7o block-copolymer known to form polymersomes. ${ }^{22}$ Using the same chain lengths would result in favourable integration of the miktoarm star into the final polymersome membrane. In order to minimise steric hindrance during the synthesis, the smaller polymer (PMPC) was attached first (Figure 2). As the central maleimide moiety absorbs light at $280 \mathrm{~nm}$ (due to the conjugated double bonds), ${ }^{38}$ and the unfunctionalised polymers do not absorb, the reactions could be monitored using HPLC with UV detection. For the reaction of PMPC-SH with dibromomaleimide, the peak corresponding to the starting molecule depleted completely and a new peak indicating the production of the functionalised PMPC/Br-Mal was observed (Figure 2). Additionally, GPC showed a shift of the polymer peak to lower molecular weight. This was expected as cleaving the disulphide bond of the initial polymer releases fragments of half the original mass (Figure 2). A similar behaviour was observed for the second substitution. HPLC analysis showed depletion of the starting molecule and the formation of a new species with GPC showing complete conversion of starting $\mathrm{PMPC} / \mathrm{Br}-\mathrm{Mal}$ and PDPA-SS-PDPA (Figure 2 and SI). The lower reactivity of the monobrominated derivative relative to the dibromo starting material, due to electronic and steric hindrance reasoning, ${ }^{39}$ prevents double substitution and thus avoids the formation of a statistical mixture. Thus, we were confident that the initial diblock pre-star PMPC-PDPA-Mal was formed successfully in high purity.

With this suitable intermediate being isolated, the last step of the star formation was evaluated. As previously mentioned, two strategies for attaching the PEG (here PEG45) were explored. Since it was derived from the more accessible maleimide precursor, the method using PEG$\mathrm{NH}_{2}$ (method a) was conducted initially (Figure 3), but we found the product to be insoluble in acidic water (GPC solvent) whilst all starting polymers were soluble in this solvent. Eventually, a GPC on chloroform/methanol was developed but it afforded inconclusive results. The refractive index detector indicated a decrease in molecular weight whilst the light scattering trace showed a partial increase (see SI for details). Hence, this method resulted in an impure molecule and was the trigger to trial the CuAAC click chemistry strategy (method b) (Figures 1,3 ). For this reaction, literature often reports the requirement for this use of a) a $\mathrm{Cu}(\mathrm{I})$ salt with suitable ligand or b) a $\mathrm{Cu}$ (II) salt together with a suitable ligand and ascorbic acid as a reducing agent. ${ }^{40}$ However, in our case, using a fresh source of $\mathrm{Cu}(\mathrm{I})$ and a reportedly suitable ligand (i.e. THPTA), ${ }^{36}$ resulted in no reaction (Figure 3 ). A complete reaction was only observed after adding ascorbic acid to the system. This suggests that acorbic acid acts as more than just a reducing agent in the reaction. GPC of the product showed a complete shift with no single PEG or residual starting diblock present (Figure 3). We thus concluded that a pure final miktoarm star was formed.

With the final miktoarm polymer isolated, its ability to self-assemble into patchy polymersome systems was investigated. To this end, several mixtures combining 
PMPC25-PDPA70 as diblock and the miktoarm as triblock were prepared: PMPC25-PDPA70 alone; with 10\% as well as $40 \%$ of the miktoarm star terpolymer, and finally the miktoarm on its own. For comparison, this study was also conducted with the impure star terpolymer originating from PEG-Amine. The morphologies of the resulting suspensions were observed by Transmission Electron Microscopy (TEM) after ten days under stirring conditions. At this stage, a mixture of diblock-copolymers (PMPCPDPA and PEG-PDPA) had previously already showed considerable demixing. ${ }^{24-26}$ To highlight the presence of surface domains, we applied our established protocol through the use of phosphostungstic acid (PTA) as a selective staining agent to image the polymersomes. ${ }^{24,25}$ PTA is known for its preferential affinity for ester groups, predominantly present in PMPC as compared to PEG (Fig. 1). This method has proven to be reliable and allowed us to proceed without further cryo-TEM imaging. The only disadvantage in the method is that the size and shape of the patches may be slightly distorted as dry TEM imaging only allows observation of collapsed vesicles. In view of this, we will only concentrate on domains if the spots are separated by further than one PMPC chain, i.e. by more than $3.5 \mathrm{~nm}^{26}$

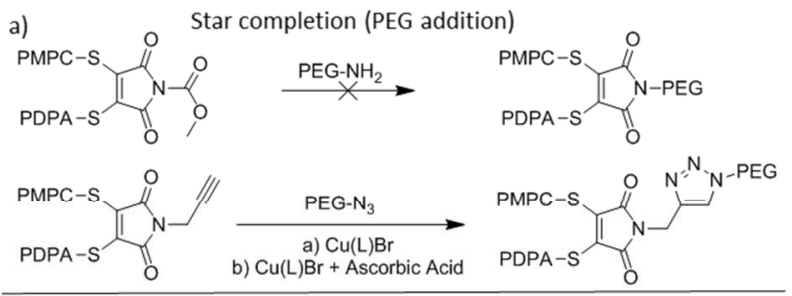

b)

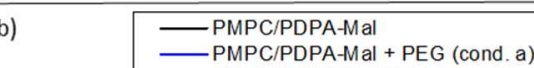
+ PEG (cond. a)

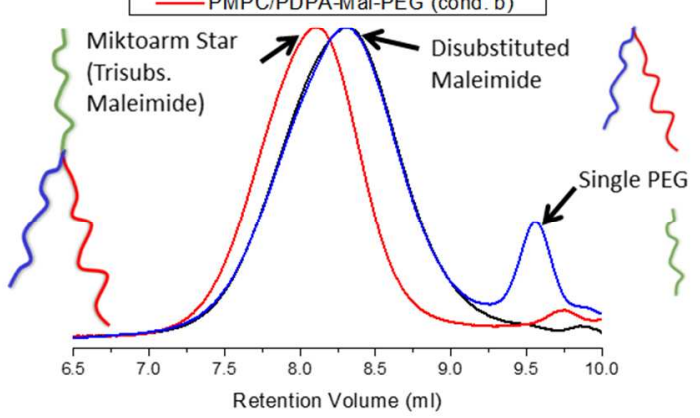

Figure 3: (a) Attaching the PEG via the amine-based strategy proved unfeasible, whilst the CuAAC was successful in conditions b). (b) GPC trace showing the presence of single PEG and no conversion of the disubstituted Maleimide for conditions a, but a full conversion of both materials for conditions $b$ ); $\mathrm{L}=$ THPTA.

As expected, no patches were present for plain PMPCPDPA polymersomes. With increasing star content, patches were formed with their composition being determined by the ratio of polymers. For $10 \%$ star content, small islets were visible as the small number of PEG chains present allowed for the formation of isolated spots of PEG. With an increasing amount, the initially formed islets interconnected with each other to develop struc- tures towards bicontinous phases. Whilst breaks between the phases were visible, larger patches of one phase were clearly visible. For the sole trifuntional miktoarm, the same amount of PMPC and PEG chains were present, but the formation of islets was not possible due to their covalent chemical linkage. This results in a bicontinous phase of PEG and PMPC respectively, on a single-chain level, hence on the edge of TEM resolution. The image represents this as a bicontinous phase of black and white areas.

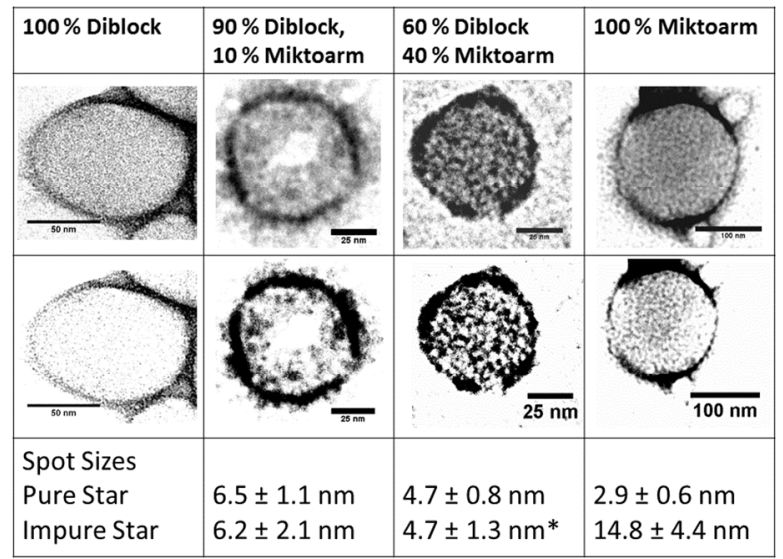

Figure 4: TEM micrographs, original and with enhanced contrast with spot sizes for polymersomes for varying PMPCPDPA diblock to PMPC/PDPA-Mal-PEG Miktoarm star polymer composition. The contrast of the images has been enhanced to emphasize the presence of patches on the vesicle surface. *On a 50/50 mixture for impure star.

With respect to the size of the domains formed, it seems logical to assume that a larger amount of lineactant could support smaller domains. In our study, the pure miktoarm follows this pattern neatly (Figure 4). An increase in miktoarm content decreases the domain size from $6.5 \mathrm{~nm}$ to $4.7 \mathrm{~nm}$ to $3 \mathrm{~nm}$. According to our previous research, a $3 \mathrm{~nm}$ size corresponds to a single PMPC chain. $^{26}$ Initially, the impure star gave a similar trend. However, we have to stress the fact that we are not certain of what exact impurities are present for the impure star. For a smaller content, the impurities do not have a great effect on the sample, but their influence grows with the amount of star polymer. One effect of the impurities is the larger error margin of domain sizes detected throughout this series. However, for the pure star, the reduction in domain size with growing star content proves that the miktoarm terpolymer has the lineactant functionality intended. Future studies will thus focus on which patterns can be achieved using our miktoarm star. This includes more polymer compositions, cellular interactions and how stable each pattern is over time.

In conclusion, we have successfully synthesised a miktoarm star terpolymer bearing three distinct arms starting from a trifunctional dibromo- $N$-propargyl-maleimide motif. PMPC and PDPA were synthesised containing a disulphide moiety which was then reduced in situ to attach it to the maleimide core. The attachment of both polymer units was monitored by HPLC as well GPC with the latter showing polymers of mono-modal distribution 
with complete conversion of starting material. The PEG was attached in the last step using the CuAAC chemistry. Much to our surprise, a specific set of conditions needed to be applied in order to get full conversion. The miktoarm star was then able to induce the formation of patchy polymersomes. Moreover, the size and shape of the patches could be regulated readily by the ratio of miktoarm star and diblock present in the formulation. In future studies, we will conduct long-term phase separation studies in order to create long-term stable patchy polymersomes for efficient drug delivery.

\section{ASSOCIATED CONTENT}

Supplementary Information (SI) including Synthetic details of all organic and polymer reactions, including NMR, HPLC, additional GPC data and TEM images of the impure star is available online This material is available free of charge via the Internet at http://pubs.acs.org.

\section{AUTHOR INFORMATION}

\section{Corresponding Authors}

* jens.gaitzsch@unibas.ch, v.chudasama@ucl.ac.uk

\section{Present Addresses}

†Imperial College London, Institute of Chemical Biology, Exhibition Road, London $\mathrm{SW}_{7}$ 2AZ, United Kingdom.

\section{Author Contributions}

The manuscript was written through contributions of all authors. All authors have given approval to the final version of the manuscript. \$These authors contributed equally. (match statement to author names with a symbol)

\section{Funding Sources}

Financial Support of the ERC (MEVIK for LM) and the DFG (Grant GA 2051/1-1 for JG) is gratefully acknowledged.

\section{ABBREVIATIONS}

ATRP (Atom Transfer Radical Polymerisation), GPC (Gel Permeation Chromatography), TEM (Transmission Electron Microscopy, HPLC (High Performance Liquid Chromatography), THPTA (Tris(3-hydroxypropyltriazolylmethyl)amine)

\section{REFERENCES}

(1) Schwille, P. Science 2011, 333, 1252.

(2) Hammer, D. A.; Kamat, N. P. FEBS Lett. 2012, 586 , 2882 .

(3) Malinova, V.; Nallani, M.; Meier, W. P.; Sinner, E. K. FEBS Lett. 2012, 586, 2146.

(4) Najer, A.; Wu, D. L.; Vasquez, D.; Palivan, C. G.; Meier, W. Nanomedicine 2013, 8, 425.

(5) Blanazs, A.; Massignani, M.; Battaglia, G.; Armes, S. P.; Ryan, A. J. Adv. Funct. Mater. 2009, 19, 2906.

(6) Lomas, H.; Du, J. Z.; Canton, I.; Madsen, J.; Warren, N.; Armes, S. P.; Lewis, A. L.; Battaglia, G. Macromol. Biosci. 2010, 10,513 .

(7) Discher, D. E.; Eisenberg, A. Science 2002, 297, 967.

(8) Gaitzsch, J.; Appelhans, D.; Janke, A.; Strempel, M.; Schwille, P.; Voit, B. Soft Matter 2014, 10, 66.

(9) Discher, D. E.; Discher, B. M.; Won, Y. Y.; Ege, D. S.; Lee, J. C. M.; Bates, F. S.; Hammer, D. A. Science 1999, $284,1143$.

(10) Messager, L.; Gaitzsch, J.; Chierico, L.; Battaglia, G. Curr. Opin. Pharmacol. 2014, 18, 104.
(11) Gaitzsch, J.; Appelhans, D.; Voit, B. Nachr. Chem. 2012 60,1176 .

(12) Cabane, E.; Malinova, V.; Meier, W. Macromol. Chem. Phys. 2010, 211, 1847.

(13) Hsu, S. P.; Chu, I. M.; Yang, J. D. J. Appl. Polym. Sci. 2012, 125, 133.

(14) Chen, X. R.; Ding, X. B.; Zheng, Z. H.; Peng, Y. X. Macromol. Rapid Commun. 2004, 25, 1575.

(15) Lomas, H.; Canton, I.; MacNeil, S.; Du, J.; Armes, S. P. Ryan, A. J.; Lewis, A. L.; Battaglia, G. Adv. Mater. 2007, 19, 4238.

(16) Gaitzsch, J.; Appelhans, D.; Grafe, D.; Schwille, P.; Voit, B. Chem. Commun. 2o11, 47, 3466.

(17) Gaitzsch, J.; Huang, X.; Voit, B. Chem. Rev. 2015, DOI: 10.1021/acs.chemrev.5boo241.

(18) Canton, I.; Battaglia, G. Chem. Soc. Rev. 2o12, 41, 2718.

(19) Photos, P. J.; Bacakova, L.; Discher, B.; Bates, F. S.; Discher, D. E. J. Controlled Release 2003, 90, 323.

(20) Cerritelli, S.; Velluto, D.; Hubbell, J. A Biomacromolecules 2007, 8, 1966.

(21) Canton, I.; Massignani, M.; Patikarnmonthon, N.; Chierico, L.; Robertson, J.; Renshaw, S. A.; Warren, N. J.; Madsen, J. P.; Armes, S. P.; Lewis, A. L.; Battaglia, G. FASEB J. 2013, 27, 98.

(22) Pearson, R. T.; Warren, N. J.; Lewis, A. L.; Armes, S. P.; Battaglia, G. Macromolecules 2013, 46, 1400.

(23) Colley, H. E.; Hearnden, V.; Avila-Olias, M.; Cecchin, D.; Canton, I.; Madsen, J.; MacNeil, S.; Warren, N.; Hu, K.; McKeating, J. A.; Armes, S. P.; Murdoch, C.; Thornhill, M. H.; Battaglia, G. Mol. Pharm. 2014, 11, 1176.

(24) Massignani, M.; LoPresti, C.; Blanazs, A.; Madsen, J.; Armes, S. P.; Lewis, A. L.; Battaglia, G. Small 2009, 5, 2424.

(25) LoPresti, C.; Massignani, M.; Fernyhough, C.; Blanazs, A.; Ryan, A. J.; Madsen, J.; Warren, N. J.; Armes, S. P.; Lewis, A. L.; Chirasatitsin, S.; Engler, A. J.; Battaglia, G. ACS Nano 2011, 5, 1775 .

(26) Ruiz-Perez, L.; Madsen, J.; Themistou, E.; Gaitzsch, J.; Messager, L.; Armes, S. P.; Battaglia, G. Polym. Chem. 2015, 6, 2065 .

(27) Battaglia, G.; Ryan, A. J. J. Am. Chem. Soc. 20o5, 127, 8757 .

(28) Stoenescu, R.; Meier, W. Chem. Commun. 2002, 3016.

(29) Li, S. T.; He, X.; Li, Q. L.; Shi, P. F.; Zhang, W. Q. ACS Macro Lett. 2014, 3, 916.

(30) Hu, H.; Liu, G. J. Macromolecules 2014, 47, 5096.

(31) Khanna, K.; Varshney, S.; Kakkar, A. Polym. Chem. 2010, $1,1171$.

(32) Steinschulte, A. A.; Schulte, B.; Erberich, M.; Borisov, O. V.; Plamper, F. A. ACS Macro Lett. 2012, 1, 504.

(33) Li, Z. B.; Kesselman, E.; Talmon, Y.; Hillmyer, M. A.; Lodge, T. P. Science 2004, 306, 98.

(34) Hadjichristidis, N.; Iatrou, H.; Pitsikalis, M.; Pispas, S.; Avgeropoulos, A. Prog. Polym. Sci. 2005, 30, 725.

(35) Castañeda, L.; Wright, Z. V. F.; Marculescu, C.; Tran, T. M.; Chudasama, V.; Maruani, A.; Hull, E. A.; Nunes, J. P. M.; Fitzmaurice, R. J.; Smith, M. E. B.; Jones, L. H.; Caddick, S.; Baker, J. R. Tetrahedron Lett. 2013, 54, 3493.

(36) Nathani, R. I.; Chudasama, V.; Ryan, C. P.; Moody, P. R.; Morgan, R. E.; Fitzmaurice, R. J.; Smith, M. E. B.; Baker, J. R.; Caddick, S. Org. Biomol. Chem. 2013, 11, 2408.

(37) Goldmann, A. S.; Glassner, M.; Inglis, A. J.; BarnerKowollik, C. Macromol. Rapid Commun. 2013, 34, 810.

(38) Robin, M. P.; Wilson, P.; Mabire, A. B.; Kiviaho, J. K.; Raymond, J. E.; Haddleton, D. M.; O'Reilly, R. K. J. Am. Chem. Soc. 2013, 135, 2875.

(39) Morgan, R. E.; Chudasama, V.; Moody, P.; Smith, M. E. B.; Caddick, S. Org. Biomol. Chem. 2015, 13, 4165. 
(40) Maruani, A.; Alom, S.; Canavelli, P.; Lee, M. T. W.; 51, 5279. Morgan, R. E.; Chudasama, V.; Caddick, S. Chem. Commun. 2o15,

1

2

3

4

5

6

7

8

9

10

11

12

13

14

15

16

17

18

19

20

21

22

23

24

25

26

27

28

29

30

31

32

33

34

35

36

37

38

39

40

41

42

43

44

45

46

47

48

49

50

51

52

53

54

55

56

57

58

59

60

TOC Image:

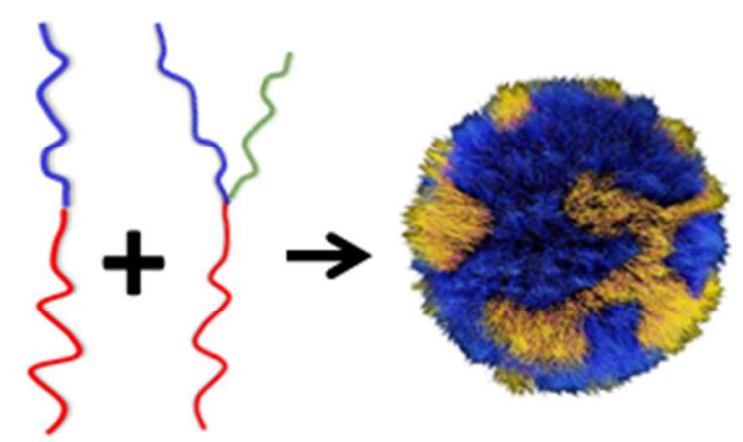

